\title{
DINE-1, the highest copy number repeats in Drosophila melanogaster are non-autonomous endonuclease-encoding rolling-circle transposable elements (Helentrons)
}

\author{
Jainy Thomas ${ }^{1}$, Komal Vadnagara ${ }^{2}$ and Ellen J Pritham $^{1 *}$
}

\begin{abstract}
Background: The Drosophila INterspersed Elements-1 (DINE-1/INE1) transposable elements (TEs) are the most abundant component of the Drosophila melanogaster genome and have been associated with functional gene duplications. DINE-1 TEs do not encode any proteins (non-autonomous) thus are moved by autonomous partners. The identity of the autonomous partners has been a mystery. They have been allied to Helitrons (rolling-circle transposons), MITEs (DNA transposons), and non-LTR retrotransposons by different authors.

Results: We report multiple lines of bioinformatic evidence that illustrate the relationship of DINE-1 like TEs to endonuclease-encoding rolling-circle TEs (Helentrons). The structural features of Helentrons are described, which resemble the organization of the non-autonomous partners, but differ significantly from canonical Helitrons. In addition to the presence of an endonuclease domain fused to the Rep/Helicase protein, Helentrons have distinct structural features. Evidence is presented that illustrates that Helentrons are widely distributed in invertebrate, fish, and fungal genomes. We describe an intermediate family from the Phytophthora infestans genome that phylogenetically groups with Helentrons but that displays Helitron structure. In addition, evidence is presented that Helentrons can capture gene fragments in a pattern reminiscent of canonical Helitrons.

Conclusions: We illustrate the relationship of DINE-1 and related TE families to autonomous partners, the Helentrons. These findings will allow their proper classification and enable a more accurate understanding of the contribution of rolling-circle transposition to the birth of new genes, gene networks, and genome composition.
\end{abstract}

Keywords: Helitron, Rolling-circle transposon, INE-1, DNAREP1, Transposable element

\section{Background}

Repetitive DNA constitutes a major portion of most multicellular eukaryotic genomes. This fraction includes tandem and interspersed repeats. Transposable elements (TEs) are the major constituent of the interspersed repetitive DNA. Class 1 retrotransposons utilize an RNA intermediate and Class 2 DNA transposons utilize a DNA intermediate as the basis for transposition (for review [1]). The RNA mediated reactions are a copy-and-paste mechanism because an RNA transcript is copied to DNA. The cut-and-paste

\footnotetext{
* Correspondence: pritham@genetics.utah.edu

'Department of Human Genetics, University of Utah, Salt Lake City, UT 84112, USA

Full list of author information is available at the end of the article
}

DNA transposons move the excised double-stranded DNA sequence of the transposon to a new target location (for review [1]). The rolling-circle transposons (Helitrons) like the retrotransposons use a copy-and-paste mechanism despite moving a DNA intermediate [2]. They are hypothesized to mobilize a single-stranded DNA molecule to a new target location [3]. Whether a TE family uses a copyand-paste versus a cut-and-paste mechanism in part influences its relative abundance in a genome (for review [1]). TEs are distinguished from other forms of repetitive DNA because they replicate via self-encoded proteins (autonomous) or by hijacking TE encoded proteins (non-autonomous) (for review [4]). Hijacking the proteins of autonomous TEs appears to be a successful strategy as 
non-autonomous TEs generally outnumber the autonomous partners. Therefore both the replication mechanism, copy versus cut-and-paste and whether or not the TE is protein-coding influence copy number.

Genome-wide analysis allows the identification of entire populations of TEs in a genome, which includes old and inactive families as well as young, active copies. The classification of autonomous TEs is generally straightforward based on the encoded protein(s), the structure of the mobile unit, and the modification of the target site (TSD). While, the non-autonomous TEs do not encode proteins, they can often be classified based on structural features and TSD. Indeed, non-autonomous TE families can be linked to autonomous partners because they share common structure and TSD, which greatly facilitates classification. However, some non-autonomous families defy classification because they lack distinctive structural features, which enable a link to be made to autonomous partners.
The Drosophila Interspersed elements (DINE-1) identified [5] on the dot chromosome and heterochromatic regions in the $D$. melanogaster genome fall into the category of non-autonomous TEs that have defied classification [6,7]. While DINE-1 elements lack coding capacity they do display well-defined structural features. The structural features include 13 bp subTIRs, a short inverted repeat (IR) (approximately 3 to 22 bp away from the 5' subTIR) and a short stem loop at the 3' end [7] (Figure 1). Analyses of target site preferences in drosophilid genomes revealed a clear insertion preference for a TT dinucleotide but were inconclusive as far as target site modification [5-7]. These analyses allowed the definition of a core DINE-1 sequence and the observation that they carry a short microsatellite sequence [7]. DINE-1 elements have also been called INE-1 [8] and DNAREP1 [9]. For the purposes of this study we refer to all related families from Drosophila as DINE-1.

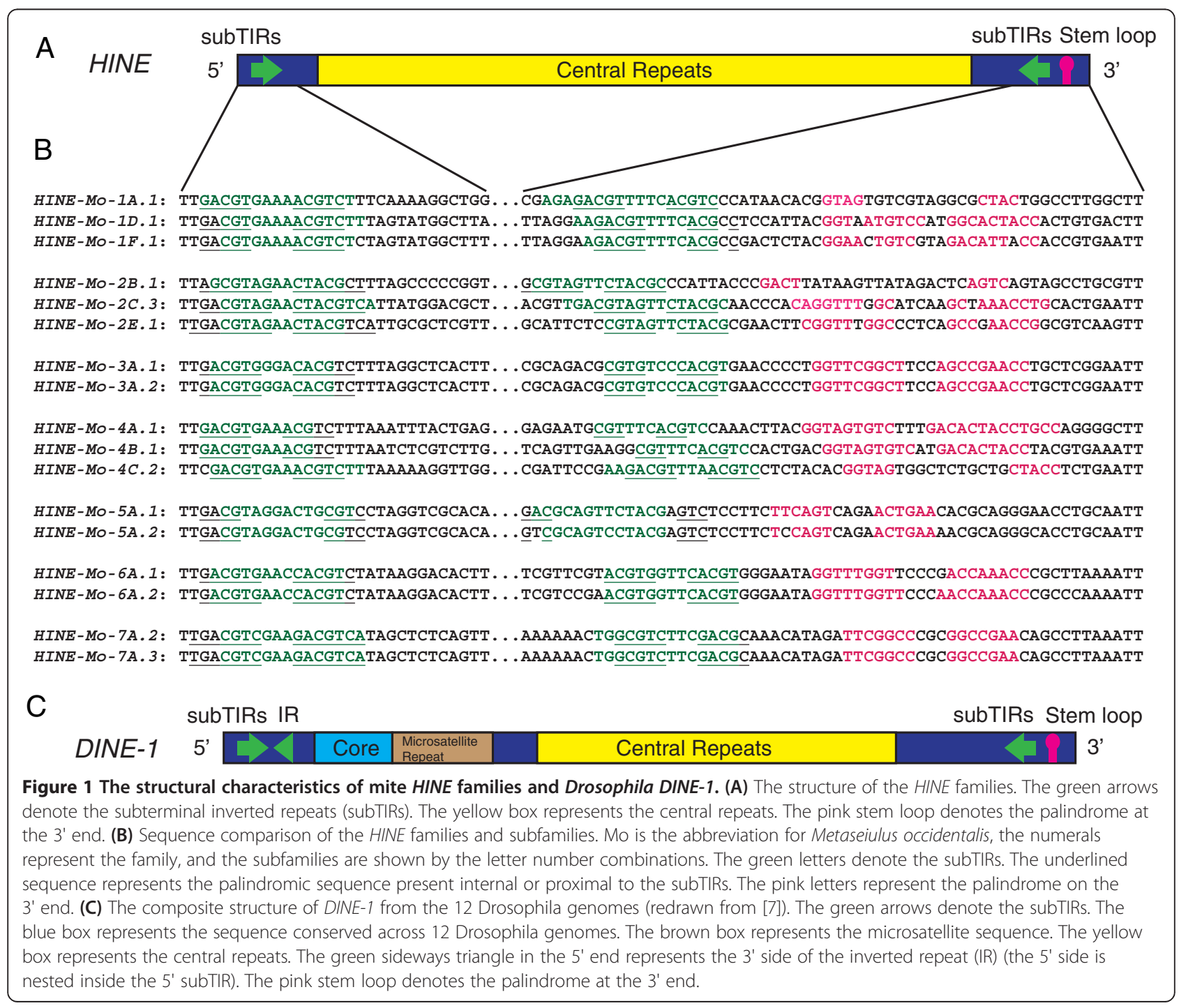


TEs with structural characteristics similar to DINE-1 (DINE-1 like) have been described in the several other genomes including several drosophilids (Additional file 1: Table S1) [10-20]. DINE-1 like elements are most abundant in the centromeres and heterochromatic regions of the chromosomes of drosophilids [5,6,10,16,21]. In $D$. serido they also predominate the heterochromatic regions of the sex chromosomes [16]. Recently active copies were found more evenly spread towards the chromosome arms and in the euchromatic regions $[6,7,10]$. Some DINE-1 like elements contain tandem repeats [16,22-24] in addition to the microsatellites that are typical of the structure [7].

Outside of Drosophila, DINE-1 like elements have been identified in many lepidopteran [25-27], hemipteran [27], and dipteran [10,28] genomes (Additional file 1: Table S1). These elements are also described from the genomes of sea urchin [24] and molluscs (Additional file 1: Table S1) [22,23,29]. DINE-1 like elements have attained high copy number in drosophilids [10] and other insect and invertebrate genomes (Additional file 1: Table S1) [22,24,25,27,28]. In D. melanogaster DINE-1 elements are the most abundant TE (for review [30]) and constitute 9.2\% (approximately $114 \mathrm{kbp}$ ) of the dot chromosome [21].

$D I N E-1$ like elements are reported to be involved in the duplication or generation of novel genes in Drosophila although whether this is direct or indirect is unclear and the mechanism remains elusive. Most of the known functional duplicates are associated with gametogenesis [31-34]. In $D$. miranda the DINE-1 like elements provide the binding sites for male specific lethal complex, which regulates dosage compensation [12,35]. In addition, DINE-1 elements are reported to provide putative transcription factor binding sites in insecticide resistance associated Cyp genes [36]. The myriad of ways that DINE-1 elements have impacted Drosophila evolution [12,31-34,36], suggests many important innovations may await discovery.

The lack of structural similarity with an autonomous partner precluded the proper classification of DINE-1 families. Indeed, these elements were initially classified as non-LTR retrotransposons perhaps because of the asymmetrical ends $[5,9,11,14]$. The interpretation of TSD creation led to the classification as the non-autonomous partners of classic cut-and-paste DNA transposons or Miniature Inverted-repeat Transposable Elements (MITEs) in some cases $[6,10,22,23,26,27]$. However, in other examples, no TSD could be readily identified and these elements were classified as Helitrons. Further support of the Helitron classification came with the identification of gene fragments with homology to the proteins encoded by some Helitrons, Helentrons and non-LTR retrotransposons $[7,25,37]$ (for review [30]). Because the structure of the DINE-1 like elements differed from canonical Helitrons [2], the relationship to Helitrons was not clear [7].
The canonical Helitrons have well defined ends (5' TC and a $3^{\prime}$ CTRR) as well as a 16 to 20 nucleotide palindrome, which is approximately $11 \mathrm{bp}$ away from the $3^{\prime}$ end [2]. They always insert between $\mathrm{A}$ and $\mathrm{T}$ nucleotides and do not create any TSD [2]. A putatively autonomous animal Helitron typically encodes a single transposase open reading frame (ORF) (Rep/Helicase) with a zincfinger, a rolling-circle motif (Rep), and a helicase domain. The plant-Helitrons encode additional ORFs related to ssDNA binding protein, Replication Protein A (RPA) (for review [30]). The non-autonomous partners of Helitrons vary in length, but share structural homology with the autonomous Helitron partners.

Recently two subtypes of Helitrons have been identified, Helentrons [38] and Helitron2 [39]. Helentrons are so called because of the presence of an apurinic/apyrimidinic (AP) endonuclease domain fused to the C-terminus of the Rep/ Helicase protein [38]. Helentrons encode additional ORFs with homology to OTU cysteine proteases [40,41] and RPA proteins [2]. The structural features of Helentrons are not known despite reports describing the coding capacity $[38,40,41]$. The Helitron2 elements have asymmetrical terminal inverted repeats and palindromic sequences on both ends. These elements encode a single ORF corresponding to the Rep/Helicase protein with no endonuclease domain [39]. However, the relationship between Helitrons, Helentrons, and Helitron 2 elements is not well understood.

Here we describe for the first time the structural features of Helentrons and describe their relationship to Helitrons and Helitron 2 elements. This analysis has allowed us to unequivocally link Helentrons to their non-autonomous partners (the DINE-1 like). Previously reported genomic impacts of DINE-1 like transposons are discussed in light of the rolling-circle transposition mechanism. This includes the involvement of DINE-1 elements in gene duplication by de novo chimeric gene assembly and the structural features that predispose co-option into regulatory networks. Presented is a new classification scheme for Helentrons and DINE-1 like families and subfamilies that take into account their sequence heterogeneity. A model of the relationship of Helentrons to Helitrons and the intermediates identified in some organisms is presented.

\section{Results}

Identification and characterization of DINE-1 like elements in the mite genome

A TE survey of the genome of the western predatory mite, Metaseiulus occidentalis lead to the identification of interspersed repeat families with defined boundaries that lack coding capacity (HINE-Mo-1-7). These repeats are characterized by 12 to 15 bp subterminal palindromic inverted repeats (subTIRs) and a 5 to $10 \mathrm{bp}$ palindrome near the 3 ' end (Figure 1A-B). The subTIRs are approximately two to four nucleotides away from the $5^{\prime}$ termini and approximately 
38-60 bp away from the 3' termini. The palindromes are approximately eight to $35 \mathrm{bp}$ of the 3 ' termini (Figure 1A-B). Some copies contain microsatellites or tandem repeats. They display an insertion preference for T-rich sequence.

HINE-Mo-(1-7) repeats share most of the structural features of the DINE-1 elements described from Drosophila, which includes the presence of subTIRs and palindromes on both ends (Figure 1C) [5-7]. Although the HINE-Mo subTIRs are themselves palindromic, a pattern not observed for the DINE-1 elements (Figure 1B, C) [7]. The $H I N E$ families differ from DINE-1 in that they do not have the IR and core sequence (Figure 1C) [7]. However, the common structural characteristics suggest that both HINE and DINE-1 belong to the same superfamily and utilize a similar mechanism of transposition.

\section{Target site modifications}

To confirm the boundary of HINE-Mo insertions, empty sites (insertion free sites) were identified (see Methods). We carefully analyzed multiple sites for each family and did not find any evidence of target site modification. HINE elements preferentially target a T-rich sequence and each insertion is flanked by at least two $\mathrm{T}$ nucleotides on each end, although it is unclear if they are distributed symmetrically (two TT on each termini) or asymmetrically (Figure 2A-C). Interestingly, in some cases elements of the same family differ in the number of $\mathrm{T}$ nucleotides on the termini, as evident from the analysis of paralogous empty sites (Figure 2D, E, Additional file 2: Figure S1, Additional file 3: Figure S2). Because these elements target $\mathrm{T}$ rich sequence, it is unclear if the Ts are distributed to only one end or both ends (Additional file 3: Figure S2). In sum, we did not find any evidence of target site modifications triggered by HINE integration, which is consistent with a single-stranded replication intermediate [3].

\section{Autonomous partner and the coding capacity}

We employed homology-based searches to identify the autonomous partners of the HINE families. Repeats could be identified that share significant sequence identity ( $84 \%$ to 99.9\%) with two subfamilies HINE-Mo-1A (Figure 3A-C) and HINE-Mo-1 K (Figure 3D-G). Pairwise alignments revealed that HINE elements are simple deletion derivatives of longer novel Helitron-like repeats that share the structural features displayed by the HINEs (Figure 3).

To determine the coding potential of the Helitron-like elements, we employed both conceptual translation of putative ORFs and homology-based searches (Blastx/conserved domain database (CDD)) [42] to the protein database. The CDD searches [42] and Blastx were employed to identify putative functional domains of the Helentron encoded proteins and related sequences in other organisms. The Helitron-like element from the mite encodes two putative proteins, which is atypical of animal Helitrons (that encode a single Rep/Helicase protein). The first corrected ORF (frame +1 ; no introns; 1,881 aa) shares $42 \%$ similarity over 1,336 aa with the Rep/Helicase/Endonuclease protein (Accession: DAA01284.1) identified from Danio rerio [38]. The alignment spanned the C-terminal endonuclease domain, which is a distinctive feature of Helentrons and is

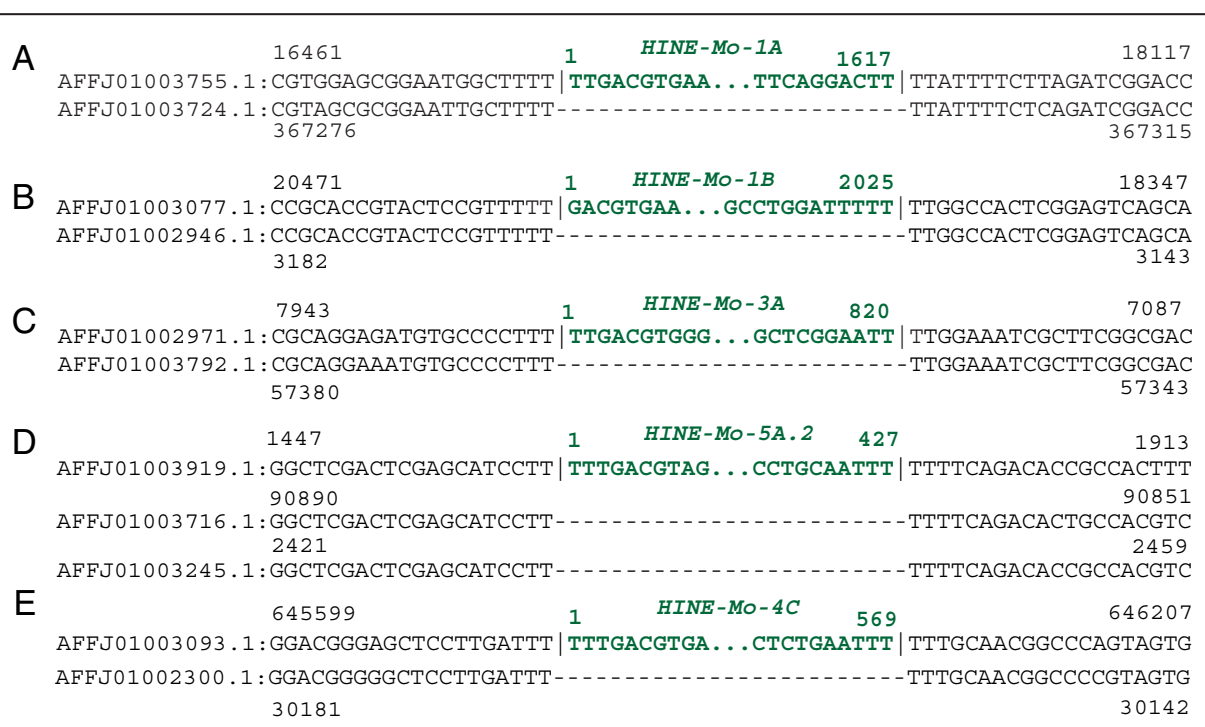

Figure 2 Comparison of the host flanking sequences of individual HINE insertions with paralogous sites in the genome that do not have the HINE insertion (empty sites). (A-E) The first line is the host sequence containing a HINE insertion (HINE-Mo-1A, HINE-Mo-1B, HINE-Mo3A, HINE-Mo-5A.2, HINE-Mo-4C) in the mite genome. The second line is a paralogous site without the HINE insertion. The sequences in black represent the host sequence and sequence in green represents the HINE sequence. The accession number and coordinates are shown in black and the length of the corresponding HINE element is shown in green. 


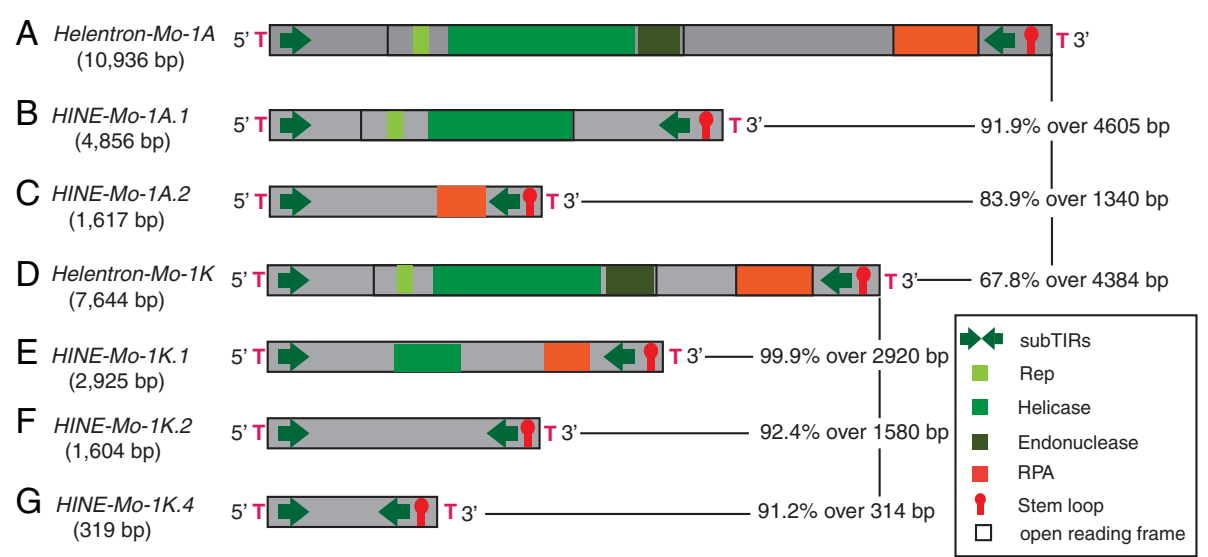

Figure 3 The structural characteristics of select mite Helentrons and their non-autonomous partners. The open black box denote the ORF encoding the Rep/Helicase protein (the lighter green is the Rep, the medium green is the helicase, and the endonuclease is the darker olive shade). The orange block denotes the ORF encoding the RPA protein. The green arrows represent the subterminal repeats (subTIRs). The red stem loop structure represents the 3' palindrome. The red T represents the flanking host sequence. Black vertical and horizontal lines point to the pairwise sequence identities and length of alignment (excluding gaps) of the sequences compared. (A) The structure of Helentron-Mo-1A. (B, C) The structures of two deletion derivatives of Helentron-Mo-1A (HINE-Mo-1A.1 and HINE-Mo-A.2). (D) Is the structure of a different subfamily of Helentrons (Helentron-Mo-1 K). (E-G) The structures of deletion derivatives related to the Helentron-Mo-1 K (HINE-Mo-1 K.1, HINE-Mo-1 K.2, and HINE-Mo-1 K.4).

not associated with canonical animal Helitrons. The closest hit was to a putative hypothetical protein identified in the Culex quinquefasciatus genome (Accession: XP_001864092.1) (61\% similarity over 1,747 aa).

Zn-finger-like motifs [38,40] are identified in the $\mathrm{N}$ terminal region of the predicted protein. The mite Rep motif shares significant sequence similarity with that of diverse Helitrons (Helitron-like, E-value: 1.62e-19, Cdd: pfam14214) [42], Helentrons, and the replication protein encoded by viruses and plasmids that utilize rolling-circle replication (RCR) (Additional file 4: Figure S3). The three conserved motifs that are necessary for the RCR $[2,38,40,43,44]$ (for review [30]) are conserved in the mite Helitron-like element (Additional file 4: Figure S3). The helicase domain identified downstream of the Rep motif has the strongest similarity with the PIF1 family belonging to the super family 1 (SF1) of helicases (PIF1-like helicase, E-value: 6.90e-19, Cdd:pfam5970) [42]. Alignment of the eight motifs that typify the PIF1 helicase family are conserved in the both the Helentrons and Helitrons (Additional file 5: Figure S4) [2]. Fused to the C-terminus of Helicase within the same ORF, the apurinic/apyrimidinic (AP) endonuclease was identified (Exo_Endo_phos_2, E-value: $1.25 \mathrm{e}-06$, Cdd:pfam14529) [42] as previously described for Helentrons [38]. The endonuclease of the mite Helitron-like element displays the characteristic seven domains described from cellular AP endonucleases, Helentrons and the non-LTR retrotransposon encoded protein (Additional file 6: Figure S5) [38,45].

The second corrected ORF (+3; no introns; 358 aa) putatively encodes the $70 \mathrm{kDa}$ subunit of the single strand (ss) binding, Replication Protein A (RPA). The
CDD searches revealed homology to the DNA binding Domains A (RPA1_DBD_A, E-value: 2.02e-15, Cdd: cd04474) and B (RPA1_DBD_B, E-value: 2.36e-12, Cdd: cd04475) [42] of RPA. Therefore the mite Helitron-like elements display all of the protein domains typical of Helentrons rather than Helitrons [38].

To further confirm that the mite Helitron-like elements belong to the Helentron group rather than Helitron group, a phylogenetic analysis (Neighbor-Joining (NJ) and maximum likelihood (ML)) was employed using an amino acid alignment of the Rep motif and eight helicase domains [46]. In this phylogenetic analysis, Helentrons (including the mite Helentron) and Helitrons form separate clades (Helentron clade bootstrap score of 95 NJ/54 ML; Helitron clade bootstrap score of $100 \mathrm{NJ} / 100 \mathrm{ML}$ ) (Additional file 4: Figure S3B). Therefore the phylogenetic analysis provides an additional line of evidence that non-autonomous families from the mite genome are Helentrons. We call the autonomous elements, Helentron-Mo. Hence the non-autonomous partners are called Helentron-associated INterspersed Elements, in short as HINEs. It is interesting to note that the Rep alignment revealed diagnostic amino acid positions that in most cases can be used to quickly distinguish between proteins encoded by Helentrons and Helitrons (Additional file 4: Figure S3A). The diagnostic positions include three amino acids ( $/ \mathrm{Y}$ w/l/y/k R) near the motif 2 '(V/I)ExQxRG(S/L)(P/L)HxH' which distinguish Helentron protein from Helitron protein. In addition, the three amino acids directly preceding the wellconserved histidine residues reveal another diagnostic site. The Helentrons have an 'S' amino acid between the $\mathrm{G}$ and $\mathrm{P}$ while the Helitrons have an $\mathrm{L}$ amino acid 
(Additional file 4: Figure S3A). These two signatures are sufficient to distinguish Helentrons from Helitrons in our sample.

\section{Association of Helentrons and HINEs in other organisms} To determine if related Helentrons were present in the organisms where DINE-1 like families had already been described or vice versa, full-length Helentrons and nonautonomous families were mined from representative species (see Methods). Two families of Helentron and their derived HINEs and a novel family of HINE (Figure 4A-D, Additional file 7: Table S2, Additional file 8) were mined from the C. quinquefasciatus genome. The Culex Helentrons display similar structural characteristics as that of the mite including the palindromic subTIRs and the stem loop at the 3' end (Additional file 7: Table S2). The elements preferentially insert within the TT dinucleotide and have a string of Ts (2 to 5 ) on the boundaries (Additional file 2: Figure S1, Additional file 3: Figure S2). These Ts are part of the element but sometime vary in number between copies (Additional file 2: Figure S1, Additional file 3: Figure S2). The non-autonomous families share $>95 \%$ sequence identity with the respective partner, Helentron.
Some copies contain simple or tandem repeats that occupy approximately $50 \%$ of the total length of the element (HINE-Cq-32A.1 and HINE-Cq-31A.1).

We investigated the genomes that are known to harbor recently amplified $D I N E-1$ s for the presence of the autonomous partner, Helentrons (D. ananassae, D. willistoni, and D. yakuba) [7]. To this end, we identified the autonomous partners of the three different DINE-1 elements described in D. ananassae, D.willistoni, and D. yakuba [7] (Additional file 8). The DINE-1s are 98\% identical with their partner Helentron and have similar structural characteristics including 13 bp subTIRs, IR, and a stem loop (Figure 4E, F, Additional file 7: Table S2). Interestingly, the Rep/Helicase/Endonuclease protein identified in the Helentron-Da-40 in D. ananassae is in the minus orientation as opposed to plus orientation found in the majority of these elements (Figure 4E). In addition, we have identified a novel family of Helentrons as well as a nonautonomous family in both $D$. willistoni and D. ananassae. This Helentron family has the palindromic subTIRs instead of the IR (Figure 4G-J). As observed earlier, these elements display an insertion preference for a TT dinucleotide, have variable number of $\mathrm{Ts}$ at their termini, and their

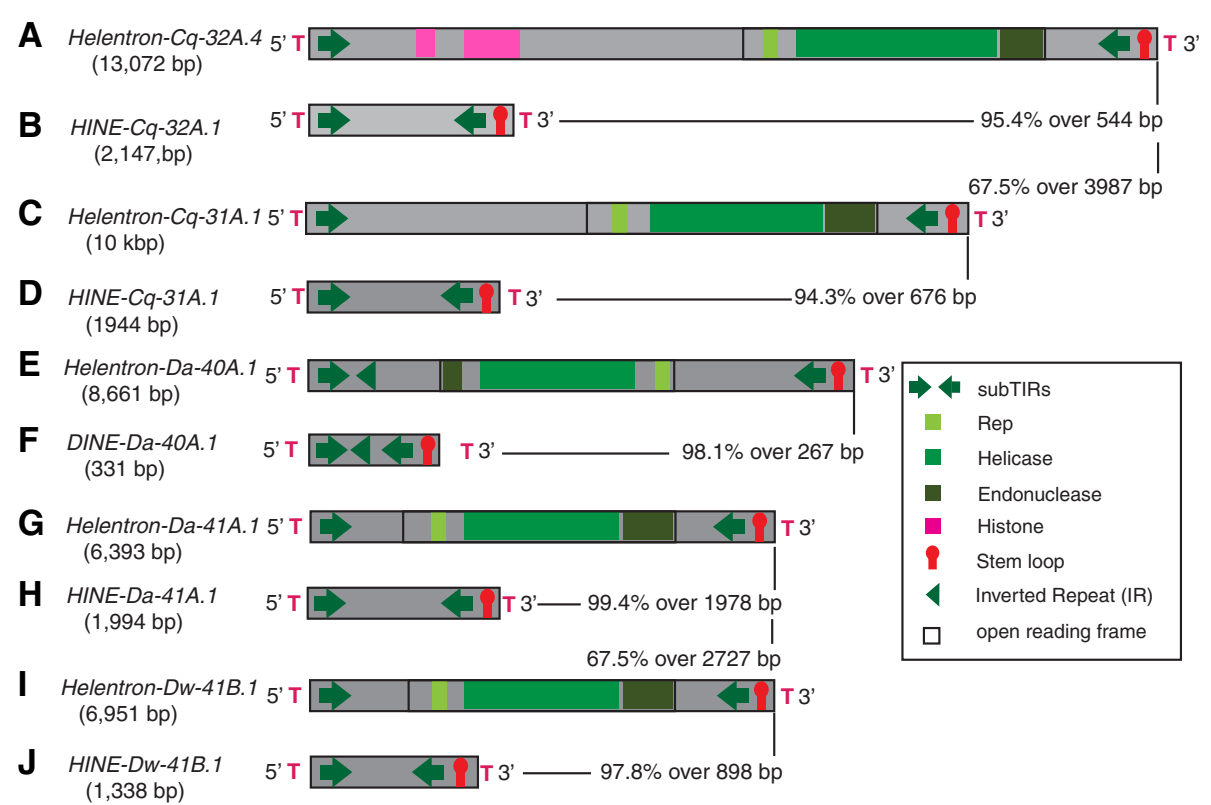

Figure 4 The structural characteristics of Helentrons and their non-autonomous partners from other species. The open black box denotes the ORF encoding the Rep/Helicase protein (the lighter green is the Rep, the medium green is the helicase, and the endonuclease is the darker olive shade). The orange block denotes the ORF encoding the RPA protein. The histone gene and gene fragments are represented in pink. The green arrows represent the subterminal repeats (subTIRs). The red stem loop structure represents the 3 ' palindrome. The green sideways triangle in the $5^{\prime}$ end represents the $3^{\prime}$ side of the inverted repeat (IR) (the $5^{\prime}$ side is nested inside the $5^{\prime}$ subTIR). The red T represents the flanking host sequence. Black vertical and horizontal lines point to the pairwise sequence identities and length of alignment (excluding gaps) of the sequences compared. (A-D) The structures of select Helentrons (Helentron-Cq-32A.4 and Helentron-Cq-31A.1) and their non-autonomous partners (HINE-Cq-32A.1 and HINE-Cq-31A.1) from the Culex quinquefasciatus genome. (E, F) The structure of another Helentron family (Helentron-Da-40A.1) from D. ananassae and the non-autonomous partner (DINE-Da-40A.1) described in [7]. The putative Rep/Helicase/Endonuclease in this Helentron is in the opposite orientation relative to others. (G-J) The structure of a novel family of Helentron (Helentron-Da-41A.1, Helentron-Dw-41B.1) present in both Drosophila ananassae and D. willistoni genomes and the non-autonomous partners (HINE-Da-41A.1, HINE-Dw-41B.1). 
boundaries were confirmed by identifying paralogous empty sites (Additional file 2: Figure S1, Additional file 3: Figure S2). The copy numbers are greater than the Helentron partner for all the non-autonomous Helentrons families across different organisms. Helentron proteins (not fulllength elements) were identified in the genomes of Strongylocentrotus purpuratus, Bombyx mori, Danaus plexippus, Rhodnius prolixus, where DINE-1 like elements were previously reported although incorrectly annotated as MITEs [24-27] (Additional file 8). These data suggest that Helentrons frequently give rise to deletion derivative/non-autonomous families.

\section{Classification of Helentrons and HINEs}

The terminal sequences including subTIRs and stem loops are shared in both Helentrons and HINEs and hence might be the signature structures necessary for the transposition. In some cases, the subTIRs are (nearly) identical (Figures 3 and 4, Additional file 7: Table S2) between two Helentron families that share $<70 \%$ identity at the nucleotide level within a species or across species (Figures 3 and 4). Due to the heterogeneity and peculiar structural features of Helitrons, a new classification criterion was established to define families and subfamilies [47]. Because Helentrons have a different structure than Helitrons, the established classification scheme does not apply. To take into account the peculiar structural features of Helentrons in relation to Helitrons, we propose a new classification scheme to identify and classify different families of Helentrons. Helentrons containing at least $11 \mathrm{bp}$ identical subTIRs are classified as members of a family (represented as the number in the name designation). Members of a subfamily share at least $80 \%$ identity over the last 60 bps of the 3 ' end (Figure 1 ) (represented as the letter following the number). The last 60 bps include the 3' subTIRs and stem loop. Using these criteria, seven families and 21 subfamilies of Helentrons and HINEs in the M. occidentalis genome (represented as Mo) were identified (Figure 1, Additional file 7: Table S2, Additional file 8). In addition, the mite genome harbors fragmented Helentrons (without ends) that diverged $(>30 \%)$ from each other. This classification scheme for Helentrons will help to understand the diversity of these families within and across genomes.

\section{Distribution of Helentrons}

We employed a homology-based search to identify autonomous Helentrons in the sequences available at the whole genome shotgun (wgs), Nucleotide collection (nr/ nt), Genome Survey Sequences (GSS), and High Through Genome Sequence (HTGS) databases. The Helentron protein queries derived by the conceptual translation of ORFs encoded by mite, Culex, Drosophila, fish, and fungi were used in Tblastn searches. The conserved amino acids of Motif two of the Rep were used as a proxy to differentiate the Helentron from Helitron proteins (Additional file 4: Figure S3). These analyses reveal the presence of Helentron proteins in many fish, Nematostella (Cnidaria), sea urchin (Echinodermata), and insects expanding the list of those previously reported (3 more insect orders (Coleoptera, Hymenoptera, Hemiptera)); 10 more families of fish; three more families from Cnidaria and Echinodermata groups (Additional file 8) [25,38,40,41] (for review [30]) (Additional file 9: Table S3). In addition we report the presence of Helentron proteins in arachnids, fungi, nematodes, molluscs, rotifer, Cephalochordata, Hemichordata, Priapulida, Annelida, lampreys, and coelacanth (Additional file 8). We have identified Helentron proteins in a range of vertebrates and invertebrates that were not known before (Additional file 8). In addition, we identify Helentron proteins in the Cotesia sesamia Mombasa bracovirus (a virus integrated in the genome of some wasps) (for review [48]). Sequences that displayed homology to Helentron-Rep proteins were identified in green algae, red algae, and Oomycete genomes (Additional file 8). Interestingly, Helentrons are not identified in most plant genomes, but a few hits were found in the databases. Because the hits were to contigs with short length and that were low copy number, we could not rule out that they were contamination (Additional file 8). Overall, Helentrons have a broad distribution in Opisthokonts (for review [49]) although absent from mammals, but are otherwise very limited in their distribution taxonomically.

\section{Identification of a Helitron-Helentron intermediate}

In our survey of the Phytophthora infestans (Oomycete) genome, a Rep/Helicase protein (1,783 aa; +2 , no introns) was identified that grouped phylogenetically with the Helentron Rep/Helicase group but did not have the endonuclease domain in the $\mathrm{C}$ terminus (Figure 5B). They have the signature amino acids in the Rep motif as observed with other Helentrons (Additional file 4: Figure S3). The structure of a full-length representative Helentron from $P$. infestans, which we call as proto-Helentron-Pi (approximately $14 \mathrm{~kb}$ ) displayed the structural features more similar to Helitrons than Helentrons (5'TT and 3' CTAG) (Figure 5). The boundary of the element was confirmed by identifying a paralogous empty site (Additional file 2 : Figure S1). None of the insertions are flanked by target site duplication (Figure 5, Additional file 2: Figure S1) and they insert between an $\mathrm{A}$ and $\mathrm{T}$ nucleotides like Helitrons (Additional file 2: Figure S1). Thus the Rep/Helicase protein is closest in amino acid similarity to Helentrons, yet the structural features of the complete elements are those of typical Helitrons (Figure 5). This suggests that Phyotophthora proto-Helentron could be an intermediate between Helitron and Helentron representing the element before the gain of the endonuclease domain.

The proto-Helentron-Pi encodes multiple putative proteins in addition to the Rep/Helicase protein. The first 


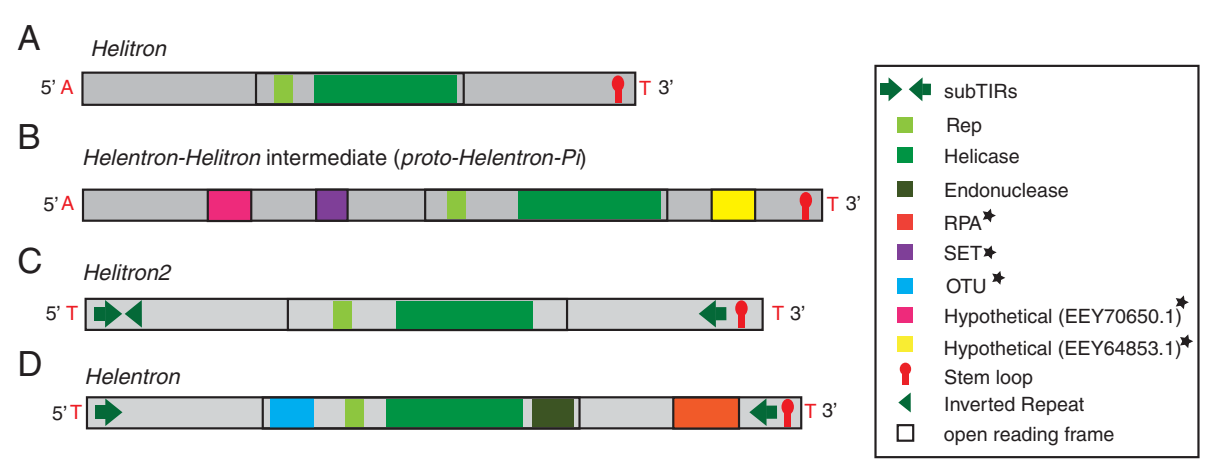

Figure 5 Comparison of the structures of a typical autonomous animal Helitron, a potential intermediate from Phytophthora infestans, Helitron2 from Chlamydomonas reinhardtii, and an autonomous Helentron family from Nematostella vectensis. (A) The structure of a representative autonomous animal Helitron. (B) The structure of the putative Helentron intermediate identified from the Phyotophthora infestans genome. (C) The structure of a Helitron2 family (redrawn from [39]). (D) The structure of an autonomous Helentron. The colored boxes represent the different encoded putative proteins or domains. The open black box denotes the ORF encoding the Rep/Helicase protein (the lighter green is the Rep, the medium green is the helicase, and the endonuclease is the darker olive shade). The orange block denotes the ORF encoding the RPA protein. The purple box represents the SET domain, blue box represents the OTU-like cysteine proteases, pink and yellow boxes represent the hypothetical proteins. Proteins and/or domains are included only if they were found in multiple families or across species. The proteins that are occasionally carried by Helentrons are indicated with a black asterisk $\left(^{*}\right)$. The green arrows denote the subterminal inverted repeats (subTIRs). The green sideways triangle in the $5^{\prime}$ end represents the $3^{\prime}$ side of the inverted repeat (IR) (the $5^{\prime}$ side is nested inside the $5^{\prime}$ subTIR). The red stem loop denotes the palindrome at the $3^{\prime}$ end. The flanking nucleotides are shown in red.

and last ORFs encode hypothetical proteins $(+2 ; 218$ aa; EEY70650.1 and -3; 266 aa; EEY64853.1). The second corrected ORF (approximately 153 aa, +3; no introns) encodes a putative SET domain (SET, E-value: 3.42e-24, Cdd:smart00317) [42] protein that has $50 \%$ similarity with the SET domain (121 aa) of histone H3 methyltransferase Clr4 of yeast, Schizosaccharomyces pombe (Accession: NP_595186.1). The characteristic features of the SET domain including the presence of variable insert region (SET-I) between the SET-N and SET-C regions (for review [50]) are present (Additional file 10: Figure S6). The highly conserved regions along with the well-conserved co-factor binding sites are present within the $\mathrm{N}$ and $\mathrm{C}$ regions (Additional file 10: Figure S6).

\section{Gene capture by Helentrons}

To determine if Helentrons capture gene fragments like Helitrons, we employed Blastx/CDD searches to identify any potential gene fragment within Helentrons and HINEs. Interestingly, Helentron-Cq-32A in the C. quinquefasciatus genome carries fragments of two histone genes (H4 superfamily E-value: 1.02e-06, Cdd:smart00428 and H15 superfamily E-value: 6.34e-05, Cdd:cd00073) [42]. Eight copies carrying these gene fragments are present in the genome (Figure 4A). The H4 gene fragment within Helentron has $84 \%$ sequence identity over 244 bp with the putative parental in the Culex genome (AAWU01017064.1|:c1090311310). Similarly, the H15 gene fragment has $83 \%$ identity over 87 bp with the putative parental in the Culex genome (AAWU01027066.1|:c14114-14203). However, both translated gene fragments contain a premature stop codon, frame shift, and indels when compared to the parental histone genes. The histone gene capture by a Helentron has not been documented in other organisms. In addition, the P. infestans proto-Helentron contains a fragment of a gene encoding a putative transmembrane protein (PITG_05761). The gene fragment within Helentron has $76 \%$ identity (1688 bp) with the putative parental gene in $P$. infestans. A short ORF (202 aa) is identified within the gene fragment in the Helentron, which contains the Ankyrin repeats (ANK, E-value: 5.20e-03, Cdd:cd00204) and the bacterial Toll-like Receptors (TIR_2) domain (TIR_2, E-value: 4.15e-15, Cdd: pfam13676) [42]. The proto-Helentron- $P i$ is amplified to 25 copies in the P. infestans genome. We did not find any potential proteincoding gene fragment within the diverse mite Helentrons and HINEs (seven families and 21 subfamilies). However, these findings suggest that Helentrons can transduce and amplify gene fragments but the frequency of capture varies between genomes, like their Helitron relatives.

\section{Discussion}

Helentrons are the autonomous partners of DINE-1 and related TEs

Our discovery of multiple autonomous Helentron families in the genome of the western predatory mite facilitated the identification of features that had not been previously defined but that are common to all endonuclease-encoding Helentrons. The structural features and target preference for T-rich sequence are shared with many nonautonomous TE families. These families known as DINE-1 like or HINEs are present in many genomes. Previously 
DINE-1 like elements have been misclassified as canonical Helitrons, MITEs, and non-LTR retrotransposons. Here we solve the mystery of DINE-1 classification by providing evidence that Helentrons are the autonomous partners (see Figures 3 and 4). Links were made between nonautonomous families and Helentrons in diverse genomes including mite, Culex, and D. ananassae, D. willistoni, and D. yakuba. Collectively, we call all non-autonomous Helentrons HINEs (for Helentron-associated interspersed elements).

\section{HINE genomic impact linked to rolling-circle transposition mechanism}

Movement by rolling-circle transposition may help explain some of the observations previously reported for HINE families, for example, high copy number in some genomes, the formation of clusters, and the association with gene duplicates. Helitrons make up a considerable fraction of the genomes of the vespertilionid bats [51], including the little brown bat (Myotis lucifugus) (Thomas et al. in revision $\mathrm{GBE}$ ) and the lepidopteran, Heliconius melpomene genome (6\% and $6.62 \%$, respectively) [52]. High genome content is consistent with a copy-and-paste transposition mechanism as is proposed for rolling-circle transposition. Clusters of Helitrons arranged in tandem arrays occur in the $M$. lucifugus genome [53] and B. mori [54] and are thought to occur when a termination signal is bypassed. In the bat and maize genome, distantly related Helitrons are also found in close proximity to other Helitron insertions sometimes directly next to one another (Thomas et al. in revision GBE), [47]. A similar pattern might be seen with Helentron mediated transposition events and might account for the clustering of related insertions $[5,16,21,22,55]$. Similarly, missed termination sequences or composite transposition between neighboring Helitrons can lead to the capture of host sequence (gene transduction). In both the bat (Thomas et al. in revision GBE) and maize (for example, [47]) genomes, thousands of non-autonomous elements carrying gene fragments have been described. Therefore, it is likely that the HINEs might also capture host sequences at some rate, Helentrons carry multiple additional functional genes that were likely captured. Indeed, many functional gene duplications have been described in association with DINE-1 elements [31-34]. It may be that the gene duplications are the result of end bypass or composite rolling-circle transposition.

\section{Captured genes useful to Helentron lifecycle}

The Rep/Helicase protein encoded by Helentrons has an endonuclease domain fused to the $\mathrm{C}$-terminus. This endonuclease is most closely related to the proteins encoded by CR1 non-LTR retrotransposons [38,41] and was either captured when a non-LTR retrotransposed into a Helitron or via a DNA-based transduction event (Thomas et al. in revision GBE). For CR1 retrotransposons target preference is likely determined by the sequence at the 3 ' end of the element [56] and is thought to be guided by the endonuclease as has been shown for other non-LTR retrotransposons [45,57-59]. In target primed reverse transcription, the endonuclease creates the single stranded nick at the target site to initiate the target primed reverse transcription $[45,60]$. We hypothesize that the target preference for the poly $\mathrm{T}$ track of the Helentrons is guided by the sequence (poly $\mathrm{T}$ or A on complementary strand) at the termini of Helentron. As observed with non-LTRs, the endonuclease may be playing a role in target preference and might be generating the single-stranded nick necessary for transposition initiation. We hypothesize that the initial nick in the $\mathrm{T}$ sequence flanking the element results in the variability of $\mathrm{T}$ nucleotides flanking the elements. Biochemical studies will be necessary to illuminate the mechanism of transposition of these elements.

We find no evidence that either Helentrons or HINEs create target site modification as has sometimes been reported in previous studies $[6,7,16,22,23,26,27]$. Analysis of the target site is made complicated by target preference for poly $\mathrm{T}$ sequences and the presence of an unknown and variable number of $\mathrm{T}$ nucleotides flanking individual elements (Figure 2, Additional file 2: Figure S1, Additional file 3: Figure S2). We propose that the variable number of $\mathrm{T}$ nucleotides at the termini of the element is the result of the endonuclease indiscriminately cleaving between $\mathrm{T}$ dinucleotides in the poly $\mathrm{T}$ stretch flanking the element for the initial single stranded cleavage.

The conservation and the probable utilization of the endonuclease for function illustrates that the capture of host genes can lead to innovation in transposition mechanism, which might have downstream consequences to the structure of the elements. Indeed, we identified an intermediate Helentron family that did not encode the endonuclease from Phytophthora (Figure 5). The Rep/Helicase encoded by this family group phylogenetically with the proteins from Helentrons (Additional file 4: Figure S3). However, the element has the structural features of a canonical Helitron and integration occurs between $\mathrm{A}$ and $\mathrm{T}$ nucleotides.

It is possible that other captured genes (Figure 5, Additional file 9: Table S3) could aid transposition. It has been proposed that the RPA encoded proteins independently captured by Helitrons in plants and Helentrons might bind single stranded DNA during transposition [2], (for review [30]). A role of the OTU-like cysteine proteases in transposition is supported by the conservation of all residues required for function and the presence of the genes in Helentron families from diverse species [40]. The protease might be involved in inducing proteolytic events involved in signaling necessary to trigger the modification of chromatin structure $[61,62]$ or in cleaving the Rep/ 
Helicase/Endonuclease subunits (for review [30]), which might facilitate transposition. Further experimental studies are necessary to understand mechanisms involved in transposition.

\section{Gene fragment transduction and duplication by Helentrons}

We looked for evidence for gene captures using homology based methods in the set of autonomous and nonautonomous elements identified in our study. Besides previously annotated examples, we only identified gene fragments in the Helentrons from the Culex and Phytophthora genomes (Additional file 9: Table S3). We did not detect gene fragments within Helentrons or HINEs as with the frequency observed in maize or bat Helitrons (>10,000 Helitrons containing gene fragments) (Thomas et al. in revision GBE) (for example, [47]. A caveat to this approach is the reliance on significant homology with known proteins. It may be that the frequency of gene captures simply varies with certain genomes, as has been observed with Helitrons [63].

Previous studies have identified non-random associations of HINE-like elements at or near the break points of several functional gene duplicates in Drosophilids [31-34]. In some of these cases, duplications occurred before as well as after speciation events. For example, for six Kep 1 gene fragment duplications, four duplications occurred before the diversification $D$. melanogaster species complex (that is, 2-3 to 6-7 million years ago) [34] while one occurred in D. melanogaster and one occurred in an ancestor of D. sechellia and D. simulans [34]. If the Kep 1 gene fragment is carried by a HINElike element and duplication occurred as the result of transposition, this pattern would suggest that Helentrons were active throughout the diversification of the $D$. melanogaster species complex. Other possible gene transduction events include CK2 $\beta$ tes and NACßtes gene duplicates also from the $D$. melanogaster species complex [33]. The $N A C \beta$ tes copies were amplified before the diversification of $D$. melangaster species complex and CK2ßtes gene duplicates have amplified in the $D$. sechellia/D. simulans lineage [33]. The hydra gene was uniquely amplified after the insertion of a HINE in the D. melanogaster species complex [31]. A burst of HINE transposition occurred in the ancestor of D. melanogaster species complex and it has been suggested that activity may have continued after diversification [64]. These duplication events do correlate well with the peak of HINE activity (approximately 4.6 million years ago) (average divergence $15.2 \% \pm 5.4$ SD) [64]. Pairwise divergence (1\% to $19 \%)$ estimates of the DINE copies in the $D$. sechellia and $D$. simulans suggest a longer potential period of activity as compared to D. melanogaster (3-19\%) [7]. Helitrons in bats have maintained activity for approximately 36 million years (Thomas et al. in revision GBE).
These findings indeed suggest that Helentron activity could play an important role in the amplification and dispersal of genic fragments and the generation of new functional gene duplicates. It maybe that Helentrons like Helitrons are capable of long periods of activity $[12,64]$.

\section{Conclusions}

In this study, we characterized the structural features of Helentrons and identify that they are different from the typical structural features of canonical Helitrons. In addition, we found that Helentrons and DINE-1 like elements share similar structural features, which unequivocally links the DINE-1 like elements as non-autonomous partners of Helentrons. Helentrons and its non-autonomous partners do not induce target site duplications upon transposition, but are flanked by variable number of Ts. We have also identified potential intermediates that have Rep/Helicase protein similar to Helentrons but share the structural characters with Helitrons. Hence our study provides a better understanding of the structure and distribution of Helitron-like elements across taxa. In addition, our studies illustrate that Helentrons are capable of gene transduction as their Helitron relatives.

\section{Methods}

Identification of HINEs and Helentrons from the mite genome and other selected genomes

Helentrons and HINEs were identified from the genome of the mite, Metaseiulus occidentalis during a de novo analysis of repeats. Repeatscout [65] was used to generate a consensus of the repeats present in three or more copies in the genome and HINEs were identified during the manual curation of these repeats. The structural characteristics of the HINE elements and Helentrons were compared and analyzed using Blast tools [66]. To identify the distribution pattern, Tblastn searches were carried out using the mite Helentron protein query against wgs, nr, GSS, and HTGS databases. Full-length Helentrons and HINEs were mined from Culex, D. willistoni, D. ananassae, and D. yakuba genomes to verify the relationship between Helentron and HINEs.

\section{Identification of open reading frames, conserved domains, and gene fragments}

The Translate (http://web.expasy.org/translate/) and ORF finder (http://www.ncbi.nlm.nih.gov/gorf/gorf.html) tools were utilized to identify Helentron encoded ORFs. The stop codons and frame shift mutations were corrected to obtain an intact the ORF, if necessary. The conserved domain database searches (CDD) [42] and Blastx were employed to identify putative functional domains of the Helentron encoded proteins and related sequences in other organisms. The low complexity filter was applied during CDD searches to avoid spurious results. Significant hits 
(e-values $<10^{-03}$ ) were further explored. To identify potential protein-coding gene fragments within Helentrons and HINEs, we employed Blastx/CDD based searches and significant (e-values $<10^{-03}$ ) hits (other than TEs) were further explored.

\section{Identification of paralogous empty sites}

To confirm the boundary of the elements, paralogous sites without the insertion (empty sites) were identified. To identify empty sites, a chimeric query constructed from $50 \mathrm{bp}$ upstream and downstream of the element was utilized for homology-based searches (Blastn) against genomes. Hits with $\geq 90 \%$ identity over $90 \%$ of the query are considered as an empty site.

\section{Alignments and phylogenetic analysis}

Alignment of the putative proteins encoded by Helentrons, Helitrons, and other bacterial plasmids or viruses that encode similar proteins were constructed employing MUSCLE (http://www.ebi.ac.uk/Tools/msa/muscle/) using default parameters. The alignments were visually refined using Genedoc (v. 2.7), [67]. Phylogenetic analysis of the Rep motifs and helicase domains was conducted using MEGA (v 5.05) [46] by constructing a NJ tree and the parameters selected were JTT matrix based model, 1,000 bootstrap replicates and pairwise deletion. A maximum likelihood analysis (JTT matrix based model, 1,000 bootstrap replicates) was also performed using MEGA (v 5.05) [46].

\section{Additional files}

Additional file 1: Table S1. Distribution and copy number of DINE-1-

like elements.

Additional file 2: Figure S1. Comparisons of the host flanking sequences of individual HINE insertions with paralogous sites in the genome that do not have the HINE insertion (empty sites). The first line is the host sequences with the Helentrons/HINE insertion. The second line is a paralogous site without the Helentron/HINE insertion. The black nucleotides represent the host sequence and underlined red nucleotides represent the transposable element. The accession and coordinates of the sequences are also given in black and the length of the transposable element is shown in red. (A-E) Empty sites of select Helentron/HINE insertions in Drosophila ananassae (HINE-Da-41A.2), D. willistoni (Helentron-Dw-41B.1), Culex quinquefasciatus (HINE-Cq-32A.1, HINE-Cq32A.2), and Phytophthora infestans (proto-Helentron-Pi).

Additional file 3: Figure S2. Comparison of the host flanking sequences of multiple HINE insertions and insertion free sites (empty sites) in the genome. The underlined sequences in red represent the HINEs and black nucleotide represent the host sequence. The accession and coordinates of the sequences are also given in black. (A) Multiple HINE-Da-41A insertions with their flanking sequences in the Drosophila ananassae genome. (B) Empty sites for each HINE-Da-41A insertion. The first line is the host sequence with the HINE-Da-41A insertion. The second line is a paralogous site without the HINE insertion. (C) Multiple HINE-Mo4C insertions with flanking sequences in the Metaseiulus occidentalis genome. (D). Empty sites for each HINE-Mo-4C insertion. The first line is the host sequence with the HINE-Mo-4C insertion. The second line is a paralogous site in the genome without the HINE insertion.
Additional file 4: Figure S3. The protein alignment of the Rep motif of representative Helentrons and Helitrons and a phylogenetic tree based on an alignment of the most conserved Rep motifs/Helicase domains. (A) An alignment of the Rep motif of Helentrons from 12 species, Helitrons from seven species and representative plasmids and viruses that utilizes rolling-circle replication (RCR). Black asterisks above the alignment denote the positions of the two histidines and two tyrosines known to be critical for catalytic activity of the RC elements. Identical residues are shaded in black and conservative changes are shaded in gray. Amino acids that distinguish Helentrons from Helitrons are boxed in red. The accession and coordinates of the different sequences used in the alignment are: Helentrons from Metaseiulus occidentalis Mite-1 (AFFJ01001714.1:C5449-8790), Mite-3 (AFFJ01002321.1:c999-4343) Culex quinquefasciatus (AAWU01024641.1:12176- 15496), platyfish Xiphophorus maculatus (ABB05534.1), fungi Mucor circinelloides (EPB86818.1), acornworm Saccoglossus kowalevskii (XP_002741052.1), Phytophthora infestans (AATU01002056.1:10099-12180), Nematostella vectensis (Helitron-1 NV [30], sea urchin Strongylocentrotus purpuratus (AAGJ04076666.1:8326-11865), Danio rerio (DAA01284.1), Frog Xenopus tropicalis (AAMC02019010.1: 25350- 33598) Drosophila willistoni (AAQB01006357.1:146323-152490), D. ananassae (AAPP01019845.1:107830112664), D. yakuba (AAEU02001960.1:C3447-10117). Helitrons from mite $M$. occidentalis (AFFJ01001759.1:1748-4869), D. ananassae (AAPP01018364.1:3376539124), Aphid Acyrthosiphon pisum (AC202211.4:97955-103017), Myotis lucifugus (AAPE02018439.1:1503-5146), Bombyx mori Helianu_Bm1 [54], Oryza sativa japonica (AAM92800.1), and Arabidopsis thaliana (AtHEL2p) [2]. SVTS, Spiroplasma plectro virus (AAF18311.2); Rep_SC, Streptomyces cyaneus plasmid (BAA34784.1); Rep_BB, Bacillus borstelensis plasmid (BAA07788.1); Rep_AA, Actinobacillus actinomycetemcomitans plasmid (AAC37125.1); Pf3, Pseudomonas aeruginosa bacteriophage (AAA88392). (B) A Neighbor-Joining (NJ) tree generated from the Rep motif and helicase domains. The bootstrap values calculated from maximum likelihood are listed before the backslash at each node and followed by bootstrap values calculated as part of the $\mathrm{NJ}$ analysis. The symbols in the tree are explained in the boxed legend.

Additional file 5: Figure S4. Protein alignment of the PIF1 helicase from Helentrons, Helitrons, and select organisms. The eight conserved motifs of the PIF1 family of helicases from Helentrons, Helitrons, yeast (P07271), baculovirus (Q9YMS4), TRAA_RHISN, Rhizobium sp (P55418.1), (P55418.1), and T4 phage (P32270). The accession and coordinates of the Helentrons and Helitrons used in the alignment are: Helentrons from Metaseiulus occidentalis Mite-1 (AFFJ01001714.1:C5449-8790), Mite-3 (AFFJ01002321.1:C999-4343) Culex quinquefasciatus (AAWU01024641.1:12176-15496), platyfish Xiphophorus maculatus (ABB05534.1), fungi Mucor circinelloides (EPB86818.1), acornworm Saccoglossus kowalevskii (XP_002741052.1), Phytophthora infestans (AATU01002161.1: 20532-18847), Nematostella vectensis (Helitron-1_NV [30], sea urchin Strongylocentrotus purpuratus (AAGJ04076666.1:8326-11865), Danio rerio (DAA01284.1), Frog Xenopus tropicalis (AAMC02019010.1: 25350- 33598), Drosophila willistoni (AAQB01006357.1:146323-152490), D. ananassae (AAPP01019845.1:107830-112664), D. yakuba (AAEU02001960.1:c3447-10117). Helitrons from Mite M. occidentalis (AFFJ01001759.1:1748-4869), D. ananassae (AAPP01018364.1:33765-39124), Aphid Acyrthosiphon pisum (AC202211.4:97955-103017), Rhodnius prolixus HeligloriaAi_Rp1 (ACPB01050589.1:10663-14351), Bombyx mori Helianu_Bm1 [54], Oryza sativa japonica (AAM92800.1), and Arabidopsis thaliana (AtHEL2p) [2].

Additional file 6: Figure S5. An alignment of the apurinic/apyrimidinic endonuclease alignment encoded by Helentrons, non-LTR retrotransposons and select cellular proteins. A protein alignment of the endonuclease domains of Helentrons from 12 species, non-LTR retrotransposons from five species, and three cellular endonucleases. The accession and coordinates of the different sequences used in the alignment are: Helentrons from Metaseiulus occidentalis Mite-1 (AFFJ01001714.1:C5449-8790), Mite-2 (AFFJ01002369.1:4460-5251) Culex quinquefasciatus (AAWU01024641.1:12176- 15496), platyfish Xiphophorus maculatus (ABB05534.1), fungi Mucor circinelloides (EPB86818.1), acornworm Saccoglossus kowalevskii (XP_002741052.1), sea urchin Strongylocentrotus purpuratus (AAGJ04076666.1:8326-11865), Danio rerio (DAA01284.1), Frog Xenopus tropicalis (AAMC02019010.1: 25350-33598), Drosophila willistoni (AAQB01006357.1:146323-152490), D. ananassae (AAPP01019845.1:107830112664), D. yakuba (AAEU02001960.1:c3447-10117). The cellular endonucleases are from Bos taurus APEX1_BOVIN (P23196.2). Homo sapiens APEX (AAB26054.1), Escherichia coli APEX3 (AAC74819.1). The non-LTR are from 
Daphnia pulex (EFX61861.1), Trypanosoma cruzi (CAB41692.1), Danio rerio (BAE46430.1), Oryzias latipes ReO_6 (BAB83841.1), Nematostella vectensis -Rex1_CR1, and C.elegans-Frodo_CR1 [38].

Additional file 7: Table S2. The subterminal inverted repeats (subTIRs) of Helentron-HINE families identified from different species.

Additional file 8: File S1. Accession number and coordinates of full length Helentrons and HINEs identified in the Metaseiulus occidentalis, Culex quinquefasciatus, Drosophila, Phytophthora genomes and Helentron proteins identified in different organisms for which the sequences are deposited in whole genome shotgun (wgs), Genome Sequence Survey (GSS), High Throughput Genome Sequence (HTGS) databases, and Nucleotide collection (nr/nt) databases.

Additional file 9: Table S3. The presence of genes in Helentron families identified in this study or previously described in the literature.

Additional file 10: Figure S6. An alignment of select SET-domain containing histone methyltransferases with the protein translation of SET encoding gene fragments carried by some Helentrons. A protein alignment of the $\mathrm{N}$ - and $\mathrm{C}$-terminal subregions of the SET domain (and SET-C, respectively) and the variable insert regions (SET-I) are shown. Identical residues are shaded in black and conservative changes are shaded in gray. Regions involved in binding to the cofactor product AdoHcy are indicated with green, and the three highly conserved sequence regions are indicated with a blue bar below the aligned sequences. The invariant tyrosine residue implicated to function as a general base for catalysis is indicated with a black star below the alignment. The insert region shows no structural conservation [50]. The various sequences used for alignment are histone $\mathrm{H} 3$ methyltransferase $\mathrm{Cl} 4$ from Schizosaccharomyces pombe (NP_595186.1), histone-lysine N-methyltransferase SUV39H1 isoform 2 from Homo sapiens (4507321:145-412), histone H3 methyltransferase DIM-5 from Neurospora crassa, (AAL35215.1), SET1 from Oryza sativa (AAK28975.1) putative histone-lysine $\mathrm{N}$-methyltransferase from Phytophthora infestans (XP_002999311.1), and Helentrons from P. cambivora (AUVH01093707.1): 299-12077), P. capsici (ADVJ01006715.11:c14578-831), and P. infestans (see Additional file 8).

\section{Abbreviations}

CDD: Conserved domain database; DINE: Drosophila interspersed element; HINE: Helentron associated interspersed element; JTT: Jones Taylor Thornton; LTR: Long terminal repeat; MITEs: Miniature inverted terminal repeat elements; ORF: Open reading frame; OTU: Ovarian tumor: RPA: Replication Protein A; SD: Standard deviation; SET: Su(var)3-9 and 'Enhancer of zeste'; TE: Transposable element; TIR: Toll-like Receptors; TSD: Target site duplication.

\section{Competing interests}

The authors declare that they have no competing interests.

\section{Authors' contributions}

JT conceived, designed, and conducted the study, and drafted the manuscript. KV participated in the study by mining the Phytophthora infestans Helentron and describing the coding capacity. EJP conceived of the study, participated in the design, and drafted the manuscript. All authors read and approved the final manuscript.

\section{Acknowledgements}

This work was supported by start-up funds from the University of Utah to EJP. The funding body has no role in design, in the collection, analysis, and interpretation of data, in the writing of the manuscript; and in the decision to submit the manuscript for publication.

\section{Author details}

'Department of Human Genetics, University of Utah, Salt Lake City, UT 84112 USA. ${ }^{2}$ Department of Cancer Biology, MD Anderson Cancer Center, Houston, TX 77054, USA.

Received: 1 April 2014 Accepted: 9 May 2014

Published: 4 June 2014

\section{References}

1. Pritham EJ: Transposable elements and factors influencing their success in eukaryotes. $J$ Hered 2009, 100:648-655.

2. Kapitonov W, Jurka J: Rolling-circle transposons in eukaryotes. Proc Natl Acad Sci U S A 2001, 98:8714-8719.

3. Feschotte C, Wessler SR: Treasures in the attic: rolling circle transposons discovered in eukaryotic genomes. Proc Natl Acad Sci U S A 2001, 98:8923-8924.

4. Feschotte C, Pritham EJ: DNA transposons and the evolution of eukaryotic genomes. Annu Rev Genet 2007, 41:331-368.

5. Locke J, Howard LT, Aippersbach N, Podemski L, Hodgetts RB: The characterization of DINE-1, a short, interspersed repetitive element present on chromosome and in the centric heterochromatin of Drosophila melanogaster. Chromosoma 1999, 108:356-366.

6. Yang HP, Hung TL, You TL, Yang TH: Genomewide comparative analysis of the highly abundant transposable element DINE-1 suggests a recent transpositional burst in Drosophila yakuba. Genetics 2006, 173:189-196.

7. Yang HP, Barbash DA: Abundant and species-specific DINE-1 transposable elements in 12 Drosophila genomes. Genome Biol 2008, 9:R39.

8. Wang J, Keightley PD, Halligan DL: Effect of divergence time and recombination rate on molecular evolution of Drosophila INE-1 transposable elements and other candidates for neutrally evolving sites. J Mol Evol 2007, 65:627-639.

9. Kapitonov W, Jurka J: Molecular paleontology of transposable elements in the Drosophila melanogaster genome. Proc Natl Acad Sci U S A 2003, 100:6569-6574.

10. Miller WJ, Nagel A, Bachmann J, Bachmann L: Evolutionary dynamics of the SGM transposon family in the Drosophila obscura species group. Mol Biol Evol 2000, 17:1597-1609.

11. Steinemann $M$, Steinemann S: A duplication including the $Y$ allele of Lcp2 and the TRIM retrotransposon at the Lcp locus on the degenerating neo-Y chromosome of Drosophila miranda: molecular structure and mechanisms by which it may have arisen. Genetics 1993, 134:497-505.

12. Ellison $C E$, Bachtrog D: Dosage compensation via transposable element mediated rewiring of a regulatory network. Science 2013, 342:846-850.

13. Hagemann S, Miller WJ, Haring E, Pinsker W: Nested insertions of short mobile sequences in Drosophila P elements. Chromosoma 1998, 107:6-16

14. Wilder J, Hollocher H: Mobile elements and the genesis of microsatellites in dipterans. Mol Biol Evol 2001, 18:384-392.

15. Vivas MV, García-Planells J, Ruiz C, Marfany G, Paricio N, Gonzàlez-Duarte R, de Frutos R: GEM, a cluster of repetitive sequences in the Drosophila subobscura genome. Gene 1999, 229:47-57.

16. Kuhn GC, Heslop-Harrison JS: Characterization and genomic organization of PERI, a repetitive DNA in the Drosophila buzzatii cluster related to DINE-1 transposable elements and highly abundant in the sex chromosomes. Cytogenet Genome Res 2011, 132:79-88.

17. Cáceres M, Ranz JM, Barbadilla A, Long M, Ruiz A: Generation of a widespread Drosophila inversion by a transposable element. Science 1999, 285:415-418.

18. Negre B, Ranz JM, Casals F, Cáceres M, Ruiz A: A new split of the Hox gene complex in Drosophila: relocation and evolution of the gene labial. Mol Biol Evol 2003, 20:2042-2054.

19. Marin I, Labrador M, Fontdevila A: The evolutionary history of Drosophila buzzatii. XXIII. High content of nonsatellite repetitive DNA in D. buzzatii and in its sibling D. koepferae. Genome 1992, 35:967-974.

20. Marín I, Fontdevila A: Evolutionary conservation and molecular characteristics of repetitive sequences of Drosophila koepferae. Heredity (Edinb) 1996, 76:355-366.

21. Slawson EE, Shaffer CD, Malone CD, Leung W, Kellmann E, Shevchek RB, Craig CA, Bloom SM, Bogenpohl J II, Dee J, Morimoto ET, Myoung J, Nett AS, Ozsolak F, Tittiger ME, Zeug A, Pardue ML, Buhler J, Mardis ER, Elgin SC: Comparison of dot chromosome sequences from D. melanogaster and D. virilis reveals an enrichment of DNA transposon sequences in heterochromatic domains. Genome Biol 2006, 7:R15.

22. Satovic E, Plohl M: Tandem repeat-containing MITEs in the clam Donax trunculus. Genome Biol Evol 2013, 5:2549-2559.

23. Gaffney PM, Pierce JC, Mackinley AG, Titchen DA, Glenn WK: Pearl, a novel family of putative transposable elements in bivalve mollusks. J Mol Evol 2003, 56:308-316. 
24. Cohen JB, Liebermann D, Kedes L: Tsp transposons: a heterogeneous family of mobile sequences in the genome of the sea urchin Strongylocentrotus purpuratus. Mol Cell Biol 1985, 5:2814-2825.

25. Coates BS, Sumerford DV, Hellmich RL, Lewis LC: A helitron-like transposon superfamily from lepidoptera disrupts (GAAA)(n) microsatellites and is responsible for flanking sequence similarity within a microsatellite family. J Mol Evol 2010, 70:275-288.

26. Coates BS, Kroemer JA, Sumerford DV, Hellmich RL: A novel class of miniature inverted repeat transposable elements (MITEs) that contain hitchhiking (GTCY)(n) microsatellites. Insect Mol Biol 2011, 20:15-27.

27. Zhang HH, Xu HE, Shen YH, Han MJ, Zhang Z: The origin and evolution of six miniature inverted-repeat transposable elements in Bombyx mori and Rhodnius prolixus. Genome Biol Evol 2013, 5:2020-2031.

28. Negre B, Simpson P: Diversity of transposable elements and repeats in a $600 \mathrm{~kb}$ region of the fly Calliphora vicina. Mob DNA 2013, 4:13.

29. Kourtidis A, Drosopoulou E, Pantzartzi CN, Chintiroglou CC, Scouras ZG: Three new satellite sequences and a mobile element found inside HSP70 introns of the Mediterranean mussel (Mytilus galloprovincialis). Genome 2006, 49:1451-1458.

30. Kapitonov W, Jurka J: Helitrons on a roll: eukaryotic rolling-circle transposons. Trends Genet 2007, 23:521-529.

31. Chen ST, Cheng HC, Barbash DA, Yang HP: Evolution of hydra, a recently evolved testis-expressed gene with nine alternative first exons in Drosophila melanogaster. PLoS Genet 2007, 3:e107.

32. Ding $Y$, Zhao L, Yang S, Jiang $Y$, Chen $Y$, Zhao R, Zhang $Y$, Zhang G, Dong Y, Yu H, Zhou Q, Wang W: A young Drosophila duplicate gene plays essential roles in spermatogenesis by regulating several Y-linked male fertility genes. PLoS Genet 2010, 6:e1001255.

33. Kogan GL, Usakin LA, Ryazansky SS, Gvozdev VA: Expansion and evolution of the X-linked testis specific multigene families in the melanogaster species subgroup. PLOS One 2012, 7:e37738.

34. Yang S, Arguello JR, Li X, Ding Y, Zhou Q, Chen Y, Zhang Y, Zhao R, Brunet $F$, Peng $L$, Long $M$, Wang W: Repetitive element-mediated recombination as a mechanism for new gene origination in Drosophila. PLOS Genet 2008, 4:e3.

35. Chuong EB, Feschotte C: Evolution: transposons up the dosage. Science 2013, 342:812-813.

36. Carareto CM, Hernandez EH, Vieira C: Genomic regions harboring insecticide resistance-associated Cyp genes are enriched by transposable element fragments carrying putative transcription factor binding sites in two sibling Drosophila species. Gene 2014, 537:93-99.

37. Kapitonov W, Jurka J: Helitrons in fruit flies. Repbase Rep 2007, 7:128.

38. Poulter RT, Goodwin TJ, Butler MI: Vertebrate helentrons and other novel Helitrons. Gene 2003, 313:201-212.

39. Bao W, Jurka J: Homologues of bacterial TnpB_IS605 are widespread in diverse eukaryotic transposable elements. Mob DNA 2013, 4:12.

40. Zhou Q, Froschauer A, Schultheis C, Schmidt C, Bienert GP, Wenning M, Dettai A, Volff JN: Helitron transposons on the sex chromosomes of the platyfish xiphophorus maculatus and their evolution in animal genomes. Zebrafish 2006, 3:39-52.

41. Cocca E, De Iorio S, Capriglione T: Identification of a novel helitron transposon in the genome of Antarctic fish. Mol Phylogenet Evol 2011, 58:439-446.

42. Marchler-Bauer A, Lu S, Anderson JB, Chitsaz F, Derbyshire MK, DeWeeseScott C, Fong JH, Geer LY, Geer RC, Gonzales NR, Gwadz M, Hurwitz DI, Jackson JD, Ke Z, Lanczycki CJ, Lu F, Marchler GH, Mullokandov M, Omelchenko MV, Robertson CL, Song JS, Thanki N, Yamashita RA, Zhang D, Zhang N, Zheng C, Bryant SH: CDD: a conserved domain database for the functional annotation of proteins. Nucleic Acids Res 2011, 39:D225-D229.

43. Ilyina TV, Koonin EV: Conserved sequence motifs in the initiator proteins for rolling circle DNA replication encoded by diverse replicons from eubacteria, eucaryotes and archaebacteria. Nucleic Acids Res 1992, 20:3279-3285.

44. Vadivukarasi T, Girish KR, Usha R: Sequence and recombination analyses of the geminivirus replication initiator protein. J Biosci 2007, 32:17-29.

45. Feng Q, Moran JV, Kazazian HH, Boeke JD: Human L1 retrotransposon encodes a conserved endonuclease required for retrotransposition. Cell 1996, 87:905-916.

46. Tamura K, Peterson D, Peterson N, Stecher G, Nei M, Kumar S: MEGA5: molecular evolutionary genetics analysis using maximum likelihood, evolutionary distance, and maximum parsimony methods. Mol Biol Evol 2011, 28:2731-2739.

47. Yang LX, Bennetzen JL: Distribution, diversity, evolution, and survival of Helitrons in the maize genome. Proc Natl Acad Sci U S A 2009, 106:19922-19927.

48. Webb B, Fisher T, Nusawardani T: The natural genetic engineering of polydnaviruses. Ann N Y Acad Sci 2009, 1178:146-156.

49. Keeling PJ, Burger G, Durnford DG, Lang BF, Lee RW, Pearlman RE, Roger AJ, Gray MW: The tree of eukaryotes. Trends Ecol Evol 2005, 20:670-676.

50. Marmorstein R: Structure of SET domain proteins: a new twist on histone methylation. Trends Biochem Sci 2003, 28:59-62.

51. Thomas J, Sorourian M, Ray D, Baker RJ, Pritham EJ: The limited distribution of Helitrons to vesper bats supports horizontal transfer. Gene 2011, 474:52-58.

52. Han MJ, Shen $Y H, X u$ MS, Liang HY, Zhang HH, Zhang Z: Identification and evolution of the silkworm helitrons and their contribution to transcripts. DNA Res 2013, 20:471-484.

53. Pritham EJ, Feschotte C: Massive amplification of rolling-circle transposons in the lineage of the bat Myotis lucifugus. Proc Natl Acad Sci U S A 2007, 104:1895-1900.

54. Thomas J, Schaack S, Pritham EJ: Pervasive horizontal transfer of rollingcircle transposons among animals. Genome Biol Evol 2010, 2:656-664.

55. Abdurashitov MA, Gonchar DA, Chernukhin VA, Tomilov VN, Tomilova JE, Schostak NG, Zatsepina OG, Zelentsova ES, Evgen'ev MB, Degtyarev SK: Medium-sized tandem repeats represent an abundant component of the Drosophila virilis genome. BMC Genomics 2013, 14:771.

56. Silva R, Burch JB: Evidence that chicken CR1 elements represent a novel family of retroposons. Mol Cell Biol 1989, 9:3563-3566.

57. Cost GJ, Boeke JD: Targeting of human retrotransposon integration is directed by the specificity of the $L 1$ endonuclease for regions of unusual DNA structure. Biochemistry 1998, 37:18081-18093.

58. Feng Q, Schumann G, Boeke JD: Retrotransposon R1Bm endonuclease cleaves the target sequence. Proc Natl Acad Sci U S A 1998, 95:2083-2088.

59. Takahashi H, Fujiwara H: Transplantation of target site specificity by swapping the endonuclease domains of two LINEs. EMBO J 2002, 21:408-417.

60. Christensen SM, Eickbush TH: R2 target-primed reverse transcription: ordered cleavage and polymerization steps by protein subunits asymmetrically bound to the target DNA. Mol Cell Biol 2005, 25:6617-6628.

61. Balakirev MY, Tcherniuk SO, Jaquinod M, Chroboczek J: Otubains: a new family of cysteine proteases in the ubiquitin pathway. EMBO Rep 2003, 4:517-522.

62. Makarova KS, Aravind L, Koonin EV: A novel superfamily of predicted cysteine proteases from eukaryotes, viruses and Chlamydia pneumoniae. Trends Biochem Sci 2000, 25:50-52.

63. Sweredoski M, DeRose-Wilson L, Gaut BS: A comparative computational analysis of nonautonomous Helitron elements between maize and rice. BMC Genomics 2008, 9:467.

64. Singh ND, Petrov DA: Rapid sequence turnover at an intergenic locus in Drosophila. Mol Biol Evol 2004, 21:670-680.

65. Price $A L$, Jones NC, Pevzner PA: De novo identification of repeat families in large genomes. Bioinformatics 2005, 21:1351-1358.

66. Altschul SF, Gish W, Miller W, Myers EW, Lipman DJ: Basic local alignment search tool. J Mol Biol 1990, 215:403-410.

67. Nicholas KB, Nicholas HB Jr, Deerfield DW II: GeneDoc: analysis and visualization of genetic variation. EMBNEWNEWS 1997, 4:14.

doi:10.1186/1759-8753-5-18

Cite this article as: Thomas et al:: DINE-1, the highest copy number repeats in Drosophila melanogaster are non-autonomous endonucleaseencoding rolling-circle transposable elements (Helentrons). Mobile DNA 2014 5:18. 